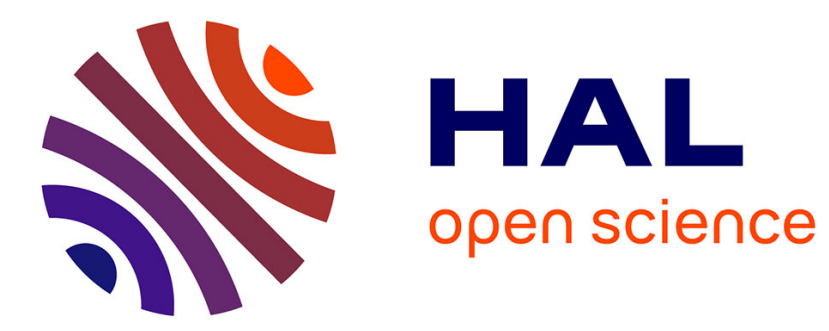

\title{
Inquisition romaine et monarchie française au XVIe siècle
}

Alain Tallon

\section{To cite this version:}

Alain Tallon. Inquisition romaine et monarchie française au XVIe siècle. Inquisition et pouvoir, 2002, Aix-en-Provence, France. p. 311-323. hal-02062896

\section{HAL Id: hal-02062896 https://hal.sorbonne-universite.fr/hal-02062896}

Submitted on 10 Mar 2019

HAL is a multi-disciplinary open access archive for the deposit and dissemination of scientific research documents, whether they are published or not. The documents may come from teaching and research institutions in France or abroad, or from public or private research centers.
L'archive ouverte pluridisciplinaire HAL, est destinée au dépôt et à la diffusion de documents scientifiques de niveau recherche, publiés ou non, émanant des établissements d'enseignement et de recherche français ou étrangers, des laboratoires publics ou privés. 


\section{INQUISITION ROMAINE ET MONARCHIE FRANÇAISE AU XVIE SIECLE}

Le 18 mai 1588 à Paris, alors que cinq jours plus tôt Henri III a fui sa capitale, chassé par les barricades, le nonce Giovanni Francesco Morosini reçoit la visite du nouveau maître de Paris, et peut-être du futur maître de la France, le duc de Guise. Conformément à la politique de Sixte Quint, hostile à la maison de Lorraine et à la Ligue, le nonce déplore "il lacrimoso accidente della ribellione di questa città"1, mais, tout en prêchant la réconciliation avec le roi il veut en même temps profiter de la victoire du parti catholique pour pousser l'avantage et obtenir satisfaction aux vieilles revendications du Saint-Siège. Sans surprise, il demande à nouveau la réception officielle des décrets du concile de Trente dans le royaume, mais, de façon plus originale, il ajoute l'introduction de l'Inquisition en France. Le duc de Guise retarde sa réponse, mais le 2 juin il vient lui même la donner au nonce : il faut retarder la demande d'introduction du concile en France, que le roi a peu de chance d'accorder - le nonce fait alors remarquer que la Ligue pose bien d'autres exigences beaucoup plus désagréables à Henri III - et il n'est pas question d'introduire en France l'Inquisition, dont le seul nom est odieux à tous les Français, même les plus catholiques ; les ennemis de la Ligue n'ont même rien trouvé de mieux pour la discréditer que de dire qu'elle établira en France l'Inquisition sur le mode espagnol. Morosini proteste en disant que l'Inquisition romaine n'est pas de même nature, mais Guise lui répond que les Français ne font pas de différence.

Étonnant dialogue, que les historiens de la Ligue n'ont pas relevé, sans doute parce qu'il ne correspond pas à l'image, qui colle encore aujourd'hui aux Guise et aux ligueurs, d'un alignement systématique sur le catholicisme romain ou espagnol. Or, Guise, en refusant de façon aussi nette l'Inquisition, qu'elle soit contrôlée par l'État ou par le Saint-Siège, se conforme à une tradition gallicane aussi ancienne que l'Inquisition romaine, tradition que l'âpreté des luttes confessionnelles ne semble pas avoir entamée. On présentera ici une première approche de cette tradition et des rapports entre royaume de France et Inquisition romaine au XVI siècle $^{2}$.

\footnotetext{
${ }^{1}$ Voir sa dépêche du 18 mai 1588 dans ASV, Nunziature di Francia 27, fol. 114, sq. et du 2 juin 1588, Ibid., fol. 130 sq. Sur l'ensemble des négociations de Morosini en mai-juin 1588, voir Victor Martin, Le gallicanisme et la Réforme catholique, Paris, 1919, p. 231 sq.

2 Je prépare une étude plus vaste sur ce sujet.
} 
La bulle Licet ab initio du 4 juillet 1542 établissait la compétence universelle du Saint-Office en matière d'hérésie, avec la réserve des inquisitions ibériques. Or, on peut reprendre toute la correspondance des nonces sous François Ier, il n'est jamais question d'établir la nouvelle juridiction dans le royaume, où subsiste, surtout dans le Midi, des tribunaux inquisitoriaux, héritages de la lutte contre les hérésies médiévales. Certes, dans les années 1530, ces tribunaux ont été discrédités soit par leur excessive rigueur en Provence, soit pour leur excessive sympathie pour les accusés à Toulouse 3 . Dans les deux cas, les parlements sont intervenus pour restreindre un peu plus la juridiction inquisitoriale. La répression de l'hérésie est en effet l'affaire du pouvoir temporel, qui s'en acquitte avec zèle. William Monter a montré de façon probante que la fin du règne de François Ier marque le point culminant de cette répression par les tribunaux laïcs.

Rome n'aurait donc pas cherché à imposer à une France au gallicanisme toujours sourcilleux une nouvelle juridiction et à courir le risque d'une épreuve de force d'autant plus inutile que la papauté n'avait rien à reprocher au zèle antihérétique du roi à l'intérieur du royaume. Si cette explication est bien sûr valable, il n'en reste pas moins que le pragmatisme manifesté par Paul III doit être souligné et éclaire les motivations du pontife : quand il crée le Saint-Office, le pape peut bien proclamer son caractère universel, il songe avant tout et même exclusivement à l'Italie. S'il poursuit, avec une ténacité capable de faire des compromis, l'introduction de l'Inquisition romaine dans les États italiens, et notamment à Venise, où la susceptibilité des autorités temporelles est comparable à celle de la France, il reste absolument silencieux vis-à-vis du roi de France. La contradiction entre les intentions universelles affichées dans la bulle Licet ab initio et la réalisation purement italienne ne vient pas d'une résistance acharnée de gallicans sourcilleux, mais de l'absence de toute initiative romaine en dehors de la péninsule pour établir l'autorité de la nouvelle congrégation.

Progressivement, cependant, le champ d'action purement italien du Saint-Office s'étend, sans que cette extension soit le résultat d'une politique cohérente et clairement assumée par les papes ou les cardinaux inquisiteurs. Mais, inévitablement, des noms français sont cités dans les dépositions de témoins ou les aveux des accusés devant le Saint-Office et la documentation est archivée, sans conduire à de véritables procédures. Les renseignements sont transmis à l'occasion au pape et à son entourage proche. En septembre 1553, Henri II nomme Jean de Monluc à l'évêché de Valence, nomination

3 William Monter, Judging the French Reformation. Heresy Trials by Sixteenth-Century 
que le nonce appuie chaleureusement ${ }^{4}$. Rome répond avec un peu de gêne que Monluc a été mis en cause par un franciscain hérétique récemment brûlé Campo dei Fiori, Fra Giovanni Buzio de Montalcino, qui a affirmé l'avoir entendu tenir des propos sacramentaires ${ }^{5}$. Même si le pape veut tout faire pour satisfaire Monluc, il craint que les cardinaux députés pour l'Inquisition ne soulèvent quelques difficultés en consistoire. La crainte de Jules III se révèle infondée puisque Monluc reçoit ses bulles d'investiture en mars 1554 .

Un changement se produit sous le pontificat de Paul IV. Pour la première fois, la papauté semble se soucier de l'organisation de la répression en France et si on peut, pour expliquer ce changement, invoquer la menace hérétique de plus en plus visible dans le royaume, il faut aussi en comprendre les raisons purement romaines : l'inquisition est pour Paul IV un moyen d'affirmer, au delà de l'Italie, le rôle prééminent de la papauté dans la répression de la dissidence religieuse, et par là, la plénitude de pouvoir dans l'Eglise que le Saint-Siège revendique avec un nouvel éclat. Il semble bien que ce soit Paul IV qui ait pris l'initiative de cette tentative d'introduire en France une forme d'inquisition centralisée. Aux archives du Saint-Office à Rome est conservé une sorte d'aide-mémoire ${ }^{6}$, intitulé "Pro offitio Sanctae Inquisitionis in Regno Franciae". On y détaille assez brièvement les lieux du royaume de France infestés par l'hérésie. Ces informations très disparates ont sans doute été extraites de divers témoignages et procès à la disposition du Saint-Office, suivant la méthode de travail habituelle à la bureaucratie inquisitoriale. Ce bref mémoire se termine sans date, ni nom de destinataire, mais il n'est pas difficile de l'identifier : le cardinal Carlo Carafa, qui part comme légat en France en mai 1556, arrive à Fontainebleau le 16 juin, et est de retour à Rome le 7 septembre ${ }^{7}$. Sa mission principale est officiellement de parachever la paix entre les princes chrétiens, juste après la trêve de Vaucelles, en réalité de négocier l'alliance entre la France et le Saint-Siège contre les Habsbourgs. Mais en marge de ces grandes manœuvres politiques, le légat doit faire valoir au roi la nécessité d'établir l'inquisition en France, et la lettre d'Henri II à son ambassadeur à Rome, Odet de Selve,

Parlements, Cambridge (Mass.)-Londres, Harvard University Press, 1999, p. 75 sq.

4 Jean Lestocquoy éd., Correspondance du nonce en France Prospero Santa Croce (15521554), Rome, Université Grégorienne, 1972, (Acta Nuntiaturae Gallicae, 9), p. 210-211, Santa Croce au cardinal del Monte, Châlis, 2 septembre 1553.

5 Ibid., p. 221, Giulo Canano à Santa Croce, Rome, 30 septembre 1553.

${ }^{6}$ ACDF, S. O., St. St., L 6-n, fol. 15-16 vº.

${ }^{7}$ Bernard Barbiche et Ségolène de Dainville-Barbiche, «Les légats a latere en France et leurs facultés aux XVI et XVII· siècles », Archivum historiae pontificae, 23, 1985, p. 157. 
datée du 13 février 1557, qui demande un bref pontifical créant des inquisiteurs en France, mentionne "les persuasions et advis que le Cardinal Caraffe estant pardeçà m'en donna de la part de nostre saint Père"8.

Rome a donc pris l'initiative de proposer l'installation du Saint-Office en France : Paul IV, en ce domaine comme en d'autres, rompt avec la politique de ses prédécesseurs, qui n'avaient jamais vraiment cherché à étendre les compétences de l'Inquisition hors d'Italie. Mais il convient de ne pas trop exagérer cette rupture, car le résultat des sollicitations pontificales est loin d'un projet initial, si tant est qu'il ait existé de façon aussi délibérée, qui aurait permis de faire reconnaître en France l'autorité de l'Inquisition romaine. Les historiens ont toujours tenu pour une évidence la parfaite identité de vue entre les deux persécuteurs, Paul IV et Henri II, sur la répression de l'hérésie et ses modalités ${ }^{9}$. On en est loin comme la lettre du roi à son ambassadeur le prouve : en raison de "quelques difficultés, alleguans ceux des Estats de mon Royaume qui ne veulent recevoir, approuver ny observer ladite Inquisition, les troubles, divisions et autres inconveniens qu'elle pouroit apporter avec soy, et mesme en ce temps de guerre, il m'a semblé pour le mieux d'y pourvoir par autre voye, et supplier le Pape, comme je veux que vous fassiez tres instamment de ma part, à ce qu'il décerne son Bref Apostolique à tel de Messieurs les Cardinaux et autres grands et notables personnages ecclésiastiques, estant par deçà que sa Sainteté advisera ; contenans ledit Bref pouvoir de subdéleguer et substituer par eux tels autres bons et vertueux Prélats, soit Evesques, ou autres constituez en dignité ecclésiastique, ou pour le moins suffisans Docteurs en la faculté de Théologie, qu'ils pourront choisir, connus et approuvé zelateurs de nostre sainte foy et religion, pour par eux et lesdits substituez et déleguez estre procedé à l'introduction et observation de ladite Inquisition, en la forme et maniere accoustumée de droit, sous l'autorité du saint Siege Apostolique, avec l'invocation du bras seculier et juridiction temporelle"10. Des cardinaux romains du Saint-Office, de leur éventuel contrôle des activités des inquisiteurs français, il n'est fait nulle mention. Henri II n'a aucunement l'intention d'établir une inquisition sous le contrôle de la congrégation romaine, pas plus qu'il n'a la possibilité d'instituer une inquisition d'État sur le modèle

${ }^{8}$ G. Ribier éd., Lettres et mémoires d'Estat des roys, princes, ambassadeurs et autres ministres sous les règnes de François premier, Henri II, et François II..., Paris, François Clouzier et veuve Aubouyn, 1666, t. II, p. 677, roi à de Odet de Selve, Paris, 13 février 1556 (a. s.).

${ }^{9}$ Voir Nicola M. Sutherland, p. 53 sq. et "Was There an Inquisition in Reformation France ?" dans Princes, politics and religion, 1547-1589, Londres, Hambledon Press, 1984, p. 13 sq.

10 G. Ribier éd., op. cit. 
espagnol. Il semble bien que ce dernier modèle ait inspiré quelques projets en 1555 , mais la ferme opposition des parlementaires, dont le président Séguier s'est fait le porte-parole en octobre 1555, a fait reculer le roi. L'inquisition française se présente sous un jour totalement inédit, différent même du compromis trouvé à Venise. Il s'agit bien d'une structure centralisée avec un ou plusieurs grands inquisiteurs, nommés par le pape. On note que, au moins dans ses premières démarches, le roi ne cherche pas à obtenir un droit de présentation ou de nomination. Cette structure centralisée n'est donc pas d'État, mais elle est nationale et entièrement autonome à l'égard de la congrégation romaine du Saint-Office. L'inquisition proposée par Henri II correspond bien aux conceptions gallicanes que la monarchie a pourtant bridées en d'autres occasions, avec le soutien de Rome.

Capable en de très rares occasions de souplesse et de pragmatisme, Paul IV accède sans discussion aux vœux du roi de France. Il propose en mars 1557 de nommer inquisiteur le cardinal de Lorraine ${ }^{11}$, auquel sont finalement adjoints les cardinaux de Bourbon et de Châtillon dans le bref du 26 avril. Le roi, par des lettres patentes du 24 juillet, prend acte de ces nominations et précise les modalités de fonctionnement de la nouvelle inquisition ${ }^{12}$. Les juges délégués par les trois cardinaux prêteront serment de fidélité devant le conseil privé. Ils devront coopérer avec les évêques. Des tribunaux d'appel seront établis par les cardinaux dans chaque ville de parlement avec dix juges, dont au moins six devront être conseillers au parlement. Le même jour, le roi signait l'édit de Compiègne, particulièrement dur puisqu'il prévoyait pour la plupart des manifestations d'hérésie la peine de mort sans possibilité d'appel. L'édit évoquait même la possibilité d'exécutions sommaires. Certes, la juridiction inquisitoriale n'était pas incompatible avec des édits antihérétiques promulgués par le pouvoir temporel, mais ici le contraste entre la tatillonne procédure inquisitoriale et celle beaucoup plus expéditive des tribunaux laïcs est accentué par le fait que le roi les met en place le même jour. Cette contradiction n'a jamais été relevée, mais elle paraît pourtant révélatrice de la place réelle, assez faible, que la nouvelle inquisition tient dans les projets répressifs du roi. Ne pouvant établir une inquisition d'État en raison de la ferme opposition des parlementaires, Henri II essaye la solution de cette inquisition gallicane, tirant son autorité du seul Saint-Siège sans dépendre de lui, mais il ne se fie véritablement qu'aux

${ }^{11}$ G. Ribier éd., op. cit., t. II, p. 684, Odet de Selve au roi, Rome, 28 (sans doute plutôt le 8) mars 1557.

12 Antoine Fontanon éd., Les édits et ordonnances des rois de France, Paris, 1611, t. 4, p. 228. 
autorités séculières pour extirper l'hérésie. L'Inquisition gallicane reste d'ailleurs sur le papier. Certes, encore en janvier 1560, François II fait demander au nouveau pape Pie IV la confirmation du rescrit apostolique que Paul IV avait adressé aux cardinaux de Lorraine, Bourbon et Châtillon pour l'inquisition et propose l'ajout d'une nouvelle clause : "que celuy desdist seigneurs trois cardinaux qui se trouvera seul à la Cour, pourra prendre et faire proceder à ladite inquisition pour l'exécution d'iceluy rescrit par ceux qu'il commettra et subdeleguera de la qualité contenue par deldit premier rescrit"13, mesure qui vise évidemment à concentrer les pouvoirs d'inquisiteur général entre les mains du cardinal de Lorraine. Mais la conjuration d'Amboise emporte ces dernières velléités d'établissement d'une juridiction inquisitoriale autonome dans le royaume.

Son échec est traditionnellement et justement expliqué par la résistance des parlements, mais il faut y ajouter deux autres facteurs : la prudence de Rome, même si elle a pu désamorcer en partie les résistances gallicanes, n'a pas donné au gouvernement royal l'impression d'un véritable soutien ; surtout, ce gouvernement royal est sensible à toutes les critiques qui peuvent se manifester contre l'établissement d'une inquisition de type moderne dans le royaume. Ces critiques prouvent la précocité d'une image négative de l'inquisition dans de larges cercles. Alors que l'antihispanisme se développe en France et que la légende noire y est accueillie rapidement, la confusion entre l'inquisition romaine et celle d'Espagne a joué un rôle important. Mais surtout, dès le règne de Henri II, la France est amenée à intervenir officiellement pour s'opposer à des procédures inquisitoriales. Ces interventions sont révélatrices de l'incompatibilité qui peut exister dans l'esprit des dirigeants français entre les principes de l'Inquisition et les traditions politiques du royaume.

La véritable épuration à laquelle Paul IV se livre au sein de la curie romaine et de l'épiscopat italien a en un premier temps peu d'échos en France. Les principales victimes, à commencer par Morone et Pole, sont des membres du parti impérial et le zèle antihérétique du pape semble au début épargner les partisans les plus notoires de la France, quand bien même leur orthodoxie est douteuse. La situation se modifie quelque peu quand, après la défaite de Saint-Quentin, le 10 août 1557, Henri II abandonne Paul

13 G. Ribier éd., op. cit., t. II, p. 841, François II au cardinal de Guise, à Rome, [janvier 1560]. Cette demande semble infirmer la révocation que Paul IV aurait faite de son bref en 1558 devant l'opposition du parlement, révocation supposée par Jean Lestocquoy éd., Correspondance des nonces en France Lenzi et Gualterio, légation du cardinal Trivulzio (1557-1561), Rome, Université Pontificale Grégorienne-Ecole française de Rome, 1977 (ANG 14), p. 111. 
IV à son sort et que celui-ci doit signer la paix avec Philippe II. Le pape n'a plus à tenir compte de son alliance avec le roi très chrétien et l'on voit apparaître, reprendre ou s'accélérer des procédures contre des amis italiens de la France, par exemple Pietro Carnesecchi, cité à comparaître à Rome en octobre 1557.

La diplomatie française n'intervient pas systématiquement, mais lors de l'automne 1557, l'ambassadeur français à Rome, Odet de Selve, demande instamment la libération d'un prisonnier, le comte de Pitigliano, francisé en Pétillan, Niccolò Orsini, emprisonné l'année précédente. Odet de Selve minimise les accusations portées contre le comte, les réduisant au fait qu'il a une concubine juive - mais ce péché ne lui paraît pas relever du Saint-Office - et qu'il a puni "des religieux qui luy estoient infideles et traistres" : mais dans ce dernier cas, "ce qu'il en avoit fait, estoit pour la conservation de son Estat et non pour heresie, ny mepris de la religion"14. Surtout, le comte est un fidèle allié de la France et Henri II l'a fait chevalier de l'ordre de Saint-Michel, ce qui lui donne le privilège d'être jugé par le chapitre de cet ordre. Les sollicitations françaises conduisent Paul IV à placer le comte en liberté surveillée en janvier 1558, mais quand Odet de Selve vient demander au pape de l'envoyer en France pour y être jugé par ses pairs, Paul IV est indigné par cette prétention à faire passer un privilège avant la juridiction pontificale, "de laquelle juridiction il n'y avoit homme vivant de quelque grande et privilégiée authorité qu'il fut, qui se peust dire exempt fut-il Empereur ou Roy ; et qu'il pouvoit priver et Empereurs et Roys de leurs empires et Royaumes sans avoir à en rendre compte qu'à Dieu”. Paul IV ajoute “qu'il n'y avoit Empereur, ny Roys s'ils estoient Chrestiens, qui ne deussent confesser qu'il estoit le Maistre, et qu'ils devoient tous prendre et recevoir la Loy de luy comme disciples et inferieurs ; et que si Dieu vouloit que les Apostres et leurs Successeurs jugeassent les Anges, à plus forte raison pouvoient-ils juger les hommes". L'ambassadeur français n'ose pas lui répondre craignant “d'estre mis à l'inquisition, nonobstant le privilege d'Ambassadeur dont il feroit peu de compte, puis qu'il méprise ceux des Roys"15. Il est important de noter qu'Odet de Selve raisonne en termes de privilèges quand Paul IV parle de juridiction.

L'incompréhension s'accroît encore un peu plus quand sous le pontificat de Pie IV, la papauté franchit une étape supplémentaire dans l'affirmation de la juridiction inquisitoriale: le Saint-Office intervient directement dans les affaires politiques et ecclésiastiques françaises, ouvrant une instruction contre la reine de Navarre, le cardinal

${ }^{14}$ G. Ribier éd., op. cit., t. II, p. 710, Selve au roi, Rome, 6 octobre 1557. 
de Châtillon et huit évêques soupçonnés d'hérésie. Ce chapitre important des relations entre la France et l'Inquisition romaine est traité dans ce volume par Elena Bonora et je me contenterai donc de rappeler l'indignation que suscite une telle procédure dans les milieux dirigeants français. Il faut aussi remarquer un décalage entre la politique curiale de Pie IV, hostile à la congrégation du Saint-Office qui a pris trop de pouvoirs sous Paul IV, et sa politique extérieure en matière d'hérésie où il cherche à imposer le primat romain par le biais de l'Inquisition. Je laisse aussi de côté la question de la juridiction du nonce en matière d'hérésie, rappelant simplement que les nonces obtiennent pendant les guerres de religion des pouvoirs spéciaux pour réconcilier les hérétiques préoccupation juridique qui n'avait jamais effleuré la papauté au temps de François Ier ou Henri II. Les nonces exercent d'ailleurs cette activité dans une semi-clandestinité pour éviter de froisser les autorités françaises.

Je veux concentrer mon propos sur les affaires qui se déroulent en Italie et qui mettent en cause des serviteurs du roi de France, car, au travers de l'indignation qu'elles suscitent en France et des dures négociations entre les représentants du roi et les autorités romaines, elles sont révélatrices de l'incompréhension radicale qui existe entre l'institution inquisitoriale et la monarchie française, incompréhension qui se manifeste dès 1557. En décembre 1570, quelques mois après la paix de Saint-Germain qui avait scandalisé le pape Pie V, un noble italien au service du roi de France, Gian Galeazzo San Severino, comte de Caiazzo, est arrêté dans le duché de Parme alors qu'il venait prendre possession du comté de Colorno qu'il avait reçu en héritage et il est livré à l'Inquisition romaine. L'accusation d'hérésie portée contre lui semble montée de toutes pièces, à la fois par ses rivaux à la cour de France, où il venait d'obtenir contre le candidat des Guise la charge de colonel général de l'infanterie italienne, et en Italie par les autres ayant droit à l'héritage de Colorno, notamment le comte Gian Battista Borromée, cousin de l'archevêque de Milan ${ }^{16}$. Cette arrestation provoque un incident diplomatique très grave entre la France et Rome.

À peine connue à la cour de France, celle-ci avait en effet déchaîné la colère du roi et de la famille royale. Catherine de Médicis peut écrire à la duchesse de Savoie, au sujet de cette affaire : "J'é grent peur que cet bonhomme de pappe à la fin par ses fays

${ }^{15}$ G. Ribier éd., op. cit., t. II, p. 715-716, Selve au roi, Rome, 8 janvier 1558.

${ }^{16}$ Les actes de son procès sont conservés aux archives du Saint-Office, S. O., St. St., S 5 - b. 
trouble toute la crestienté" 17 . Avec fermeté, Charles IX et Catherine de Médicis, mais aussi Monsieur, chef de la faction catholique de la cour, se disent convaincus de l'innocence de San Severino. Une partie de bras de fer diplomatique commence alors entre Rome et Paris. À Rome, le cardinal de Rambouillet, représentant du roi, demande une audience au pape et lui explique la gravité de la situation. Visiblement, les autorités françaises peinent à croire que le comte a été arrêté seulement pour hérésie et subodorent une affaire politique. Rambouillet cherche ainsi à disculper San Severino de tout projet belliqueux. Si le comte s'est rendu dans le marquisat de Saluces pour le service du roi "et non comme quelques ungs avoient malheureusement et meschamment controuvé, pour brouiller le monde, ny faire aulcune sorte d'entreprise qui peult preiudicier à personne, ny au repos de 1'Italie"18. Charles IX et Catherine de Médicis auraient donc pu comprendre l'inquiétude du souverain pontife s'imaginant une mission secrète de San Severino à Saluces. Mais cette idée n'a aucunement traversé l'esprit de Pie V: le pape reste sur le terrain purement inquisitorial et s'émerveille de voir le cardinal intervenir en faveur d'un suspect d'hérésie. Non seulement le roi ne doit pas protéger quelqu'un qui est prisonnier de l'Inquisition, mais il devrait envoyer de Paris ou de n'importe quel endroit de son royaume toute personne réclamée par elle. Le comte est poursuivi seulement pour hérésie ; il a été pris non à Saluces, mais dans les États de l'Église. Il n'est pas question de le libérer et le pape accepte tout juste de discuter de son cas lors de la prochaine réunion des cardinaux députés au Saint-Office. Pie V a cependant pris la mesure de l'indignation française, puisqu'il décide d'envoyer un nonce extraordinaire, Antonio Maria Salviati, pour tout expliquer au roi ${ }^{19}$. Au même moment, Charles IX envoie à Rome le marquis de Saint-Gouard pour exiger la libération immédiate de San Severino ${ }^{20}$. Ces deux missions extraordinaires se croisant montrent bien l'importance accordée à cette affaire dans les deux cours.

\footnotetext{
17 Catherine de Médicis, Lettres, Paris, Imprimerie Nationale, 1880, t. 4, p. 22, lettre de VillersCoutré, 1 er janvier 1571.

18 BNF, Fr. 16039, fol. 315 r $^{\circ}-v^{\circ}$, Rambouillet au roi, Rome, 25 janvier 1571.

${ }^{19}$ BNF, Fr. 16039, fol. 323, Rambouillet au roi, Rome, 26 janvier 1571 et fol. 326, Rambouillet au roi, Rome, 4 février 1571.

${ }^{20}$ Guy de Bremond d'Ars, Le père de Madame de Rambouillet. Jean de Vivonne. Sa vie et ses ambassades près de Philippe II et à la cour de Rome d'après des documents inédits. Paris, Plon, 1885, p. 27 sq. Il faut cependant se méfier de la présentation de l'affaire par Bremond d'Ars, qui attribue à son héros un succès imédiat quand au contraire il dut repartir de Rome avant la fin de l'affaire.
} 
D'après ses instructions, datées du 5 février $1571^{21}$, Salviati devait rester ferme sur le principe : San Severino était soupçonné d'hérésie et le Saint-Office était donc dans son droit en l'arrêtant. Cela faisait plus de quatre ans que l'on avait dénoncé à l'Inquisition des faits graves au sujet du comte de Caiazzo. En aucun cas, le pape n'a fait procéder à cette arrestation pour satisfaire des haines privées ou des intérêts personnels. Il ne s'est pas plus inquiété d'éventuelles intrigues du comte à Saluces. Son seul souci a été d'empêcher tout progrès de l'hérésie. San Severino est de plus vassal du Saint-Siège ; il a été pris dans le duché de Parme, fief de l'État pontifical. Le roi n'a donc pas à se sentir offensé et il doit cesser d'écouter les conseils de ceux qui veulent le brouiller avec le Saint-Siège pour se faire bien voir en Allemagne et y poursuivre des chimères politiques - claire allusion au rêve impérial de Charles IX, que Pie V moque cruellement, rappelant les échecs de François Ier et de Henri II. Le pape prie le roi de cesser ses instances, car il ne peut le satisfaire quand l'intégrité de la foi est en jeu.

Toute la ligne de défense de Pie V tient dans ces instructions, mais la grande faiblesse du pape vient justement de l'aspect relativement vide du dossier, malgré les affirmations des instructions données à Salviati. De son côté, Charles IX manifeste la même intransigeance sur les principes : le comte de Caiazzo est l'un de ses officiers, chevalier de son ordre. Il est couvert par la même protection que tous les sujets du roi de France, qui ne relèvent pas de la juridiction inquisitoriale. Dans son cas particulier s'ajoute une offense personnelle au roi, qui vient d'honorer celui qu'on arrête pour hérésie de la charge de colonel général de l'infanterie italienne. Enfin, Charles IX est persuadé que l'Espagne est derrière toute cette affaire, pour amoindrir encore le prestige français à Rome et en Italie. Cette obsession du complot espagnol, ici infondée, explique encore mieux son intransigeance. Finalement, après quelques mois de négociations, Caiazzo est assigné à résidence à San Pietro in Montorio en juillet, "sans aulcune garde aultre que sa parole"22. C'est seulement le 20 septembre 1571 que le cardinal peut enfin annoncer au roi "la plaine et entière deslivrance du Conte de Gaiasse"23. Il a été lavé de toute accusation. La persécution de ses ennemis, dont San Severino rendra compte lui-même au roi, a été telle qu'il n'aurait jamais été délivré sans

21 Sur la mission de Salviati, voir Olivier Poncet éd., "Correspondance d'Antonio Maria Salviati, nonce extraordinaire en France (1570-1571, 1571-1572)", à paraître. Je remercie très vivement Olivier Poncet de m'avoir communiqué ses transcriptions avant la parution de son édition.

22 BNF, Fr. 16039, fol. 384, Rambouillet à Villeroy, Rome, 2 juillet 1571.

23 Ibid., fol. 425. 
l'intervention de la France. Le comte va aller baiser les pieds du pape et repartira ensuite en France.

L'affaire San Severino est révélatrice des rapports entre monarchie française et Inquisition romaine : elle prouve tout d'abord le refus de la monarchie de placer ces rapports sur le terrain religieux. Dès qu'il s' agit d'inquisition, son vocabulaire devient avant tout juridique, niant toute compétence à un tribunal assimilé à tous les autres tribunaux romains dont la tradition gallicane exempte les sujets du roi. Les prétentions universelles du Saint-Office sont mises sur le même plan que tous les autres empiètements romains sur les privilèges des pouvoirs temporels. À cette continuité gallicane, la papauté tente bien d'opposer l'urgence et la radicalité de la coupure confessionnelle. Mais même Pie V, comme avant lui son mentor Paul IV, se plie finalement aux exigences françaises. On ne peut que constater ici la force du réflexe diplomatique, inhérent à la papauté moderne malgré les crispations confessionnelles.

Un autre exemple d'intervention française, cette fois auprès de la République de Venise, confirme les enseignements que l'on peut tirer de l'affaire San Severino. En 1581, Aurelio Vergerio, petit-neveu du célèbre exilé Pier Paolo Vergerio ${ }^{24}$, est arrêté sur le territoire vénitien et aussitôt réclamé par la congrégation romaine du Saint-Office. Or, celui que les Français appellent le chevalier Vergerius a accompli plusieurs missions en Allemagne au service du roi de France. Cela suffit pour que Henri III comme Catherine de Médicis interviennent en sa faveur : pendant un an, ils chargent à plusieurs reprise l'ambassadeur français à Venise, Arnaud Du Ferrier, d'obtenir sa libération ${ }^{25}$. De fait, si Vergerio est condamné à mort, il n'est pas exécuté et réussit à s'enfuir. Du Ferrier n'hésite pas à s'attribuer le mérite de cette heureuse issue, tout en insistant volontiers auprès de ses maîtres sur les difficultés rencontrées et le pouvoir exorbitant de l'inquisition.

L'ambassadeur français insiste sur l'innocence de Vergerio, mais son antipathie ouverte pour l'orthodoxie tridentine n'en fait un juge très fiable, au moins selon les critères de l'inquisition. Surtout, Du Ferrier a cette phrase révélatrice : "Quand ledict Vergerius seroit le plus grand luthérien du monde, néantmoins s'en estant Vostre

24 Andrea Del Col, «I contatti di Pier Paolo Vergerio con i parenti e gli amici italiani dopo l'esilio », Pier Paolo Vergerio il Giovane, un polemista attraverso l'Europa del Cinquecento. Convegno internazionale di studi (Cividale del Friuli, 15-16 ottobre 1998), a cura di Ugo Rozzo, Udine, Forum, 2000, p. 53-82.

25 Voir quelques exemples dans Jacqueline Boucher éd., Lettres de Henri III, roi de France, Paris, Honoré Champion, 2000, p. 211, 214, 239-40, 275, 300, 307, 308. 
Majesté et vos prédécesseurs Roys servis si longuement pour la défense et conservation de l'Eglise catholique, comme ont faits les autres Roys et Princes, mesmes les Papes qui se sont quelques fois servis des Infidèles pour la conservation de leurs Estats, ledict Vergerius meritoit d'estre mis du tout hors de cette peine" ${ }^{26}$. Le service du roi très chrétien vaut brevet d'orthodoxie, comme il peut en d'autres occasions procurer un brevet de noblesse. Arnaud $\mathrm{Du}$ Ferrier invente en quelque sorte la savonnette à hérétique.

Les interventions de la diplomatie française se font en effet seulement en faveur de serviteurs du roi mis en cause par l'Inquisition romaine, et pas en faveur de tous les sujets français. Il y a là une notable différence avec l'Espagne où les interventions de l'ambassadeur français concernent aussi des marchands ou des immigrés français installés dans la péninsule. Ce n'est pas le cas en Italie alors que des Français des mêmes catégories sociales peuvent être victimes de l'Inquisition : en Espagne, le roi a le souci d'affirmer sa juridiction exclusive sur ses sujets en matière de délit religieux, souci qu'on ne retrouve pas exactement exprimé de la même façon face au pape. Les interventions françaises en faveur de serviteurs du roi mis en cause par l'Inquisition romaine relèvent donc plus du refus de la conception que la papauté a de sa juridiction universelle que de la juridiction universelle elle-même.

Ce qui oppose radicalement la société française dans son ensemble, y compris ses éléments les plus dévoués à la cause catholique et à l'extermination des hérétiques, à l'Inquisition romaine n'est donc pas avant tout la supériorité du temporel ou l'indépendance gallicane face à Rome. L'un comme l'autre auraient pu être garantis par une inquisition de type ibérique ou vénitien. Ce qui dresse une barrière infranchissable à l'introduction de l'Inquisition, ce qui provoque les interventions royales en faveur des serviteurs de la monarchie, c'est l'attachement au privilège. Une procédure égalitaire, traitant tout justiciable de la même façon, est incompatible avec les conceptions politiques et sociales les plus ancrées dans le royaume. Cette incompatibilité radicale se manifeste dans le souci français de protéger les sujets ou les clients du roi, non du fanatisme religieux, mais de l'égalitarisme judiciaire. Rome a su répondre avec beaucoup de pragmatisme à cette demande. Les papes les plus intransigeants ont accepté des concessions diplomatiques, tout en continuant d'affirmer pour le principe le caractère universel de la juridiction inquisitoriale. Comme beaucoup d'autres, l'exemple

${ }^{26}$ BNF, Cinq Cents Colbert 368, p. 438, lettre au roi, Venise, 27 avril 1582. 
français montre que la capacité de résistance des sociétés d'Ancien Régime à l'inquisition réside avant tout dans leur conservatisme foncier et des traditions profondément ancrées que, au moins en théorie, le projet inquisitorial menaçait par son radicalisme.

Alain Tallon
Université de Paris-Sorbonne Paris IV. 